\title{
SOMATIC GENOMIC CHANGES IN THE FORMATION OF DIFFERENTIATED THYROID CARCINOMA
}

\author{
K. Vidinov ${ }^{1}$, R. Dodova ${ }^{2}$, I. Dimitrova', A. Mitkova², A. Shinkov', R. Kaneva² ${ }^{2}$ R. Kovacheva ${ }^{1}$ \\ ${ }^{1}$ Department of Endocrinology, Medical Faculty, Medical University - Sofia, Bulgaria \\ ${ }^{2}$ Molecular Medicine Center, Department of Medical Chemistry and Biochemistry, Medical Faculty, \\ Medical University - Sofia, Bulgaria
}

\begin{abstract}
Globally, the diffuse goiter affects more than $10 \%$ of the population and in some regions is endemic. Thyroid nodules are found in approximately $5 \%$ of the population using the oldest method for thyroid examination - palpation. When performing ultrasound screening, this percentage increases significantly and reaches between 20 and $75 \%$ of the total population. Thyroid carcinoma is a rare malignancy and accounts for up to $1 \%$ of all malignant tumors. It is the most common endocrine cancer and is clinically manifested as a thyroid nodule. Somatic mutations play an important role in its development. Differentiation of benign and malignant thyroid nodules is of great importance due to the different therapeutic approach. Therefore, new diagnostic tools are sought to help distinguish the two. Despite the progress in our knowledge of carcinogenesis in recent years, a number of key issues still remain unanswered. The establishment of new rare somatic mutations can improve pre-surgical diagnosis and optimize post-operative strategies for the treatment of thyroid carcinoma. Next-generation sequencing (NGS) allows for extensive mutation and genome rearrangements tracking. The results obtained with NGS provide the basis for the development of new approach for systematic genetic screening, at prevention, early diagnosis, accurate prognosis, and targeted therapy of this disorder.
\end{abstract}

Key words: differentiated thyroid carcinoma, nodular goiter, somatic mutations, next-generation sequencing (NGS)

Corresponding author: Kalin Vidinov, MD, PhD, Department of Endocrine Surgery, CCEG, Medical University - Sofia, University Hospital "Acad. Ivan Penchev", 2 Zdrave Str., 1463 Sofia, Bulgaria, tel: 8956063, e-mail: kalin_vi@hotmail.com

T hyroid cancer originates from two distinct cell types - follicular and para-follicular cells (Ccells). Thyroid carcinoma of follicular origin leads to several cancer subtypes - papillary thyroid carcinoma (PTC), follicular thyroid carcinoma (FTC), oncocytic (Hürthle) thyroid carcinoma, low-differentiated thyroid carcinoma and anaplaistic thyroid cancer. The medullary thyroid carcinoma originates from C-cells. In clinical practice, thyroid carcinomas of follicular cell origin are known as non-medullary thyroid tumors, and thyroid carcinoma originating from $C$ cells is classified as medullary carcinoma. Both non-medullary and medullary tumors of the thyroid gland can be sporadic or inherited. Hereditary non-medullary thyroid cancer, historically known as familial non-medullary thyroid cancer (FNMTC), is a rare, autosomal dominant disease that is not as well defined as familial medullary thyroid carcinoma [1]. The term FNMTC is historically incorrect and it is better to call the disease a hereditary non-medullar 
thyroid cancer. For example, Jindrichova S et al. consider that the term "familial cancer" refers to cancer that occurs in a family but without a known Mendelian model of inheritance, while "hereditary cancer" is one that either has a known genetic cause and/or shows a Mendelian model of inheritance [2]. There is considerable evidence that FNMTC shows a Mendelian model of inheritance. FNMTC is a heterogeneous disease that comprises of more than one histologic type of thyroid carcinoma, including familial papillary thyroid carcinoma (FPTC), familial follicular thyroid carcinoma (FFTC), and familial oncocytic carcinoma (Hürthle cell). The family with FNMTC may have members affected by FPTC and FFTC, as well as other rare subtypes of thyroid cancer of follicular cell origin [3]. It is also believed that FNMTC is associated with benign thyroid disease (nodular goiter) and some data suggest that it is slightly more aggressive than sporadic thyroid cancer of follicular cell origin. Genetically, FNMTC is probably a heterogeneous disease, but there are currently no genetic loci known to be responsible for most cases of FNMTC. Unfortunately, the published studies on FNTMC are scarce, often underestimated, and the studied groups - quite heterogeneous [4]. One of the main reasons for this is because most cases with FNMTC cannot be distinguished histologically from sporadic thyroid cancer, there is no specific genetic locus and no easily recognizable phenotype.

Over the last decade, our understanding of thyroid oncogenesis has developed rapidly. This is due to significant technological advances and the widespread use of high-performance molecular biological assays for changes in specific genes, miRNAs, tyrosine kinase deregulations, and more. Huge amounts of data have been generated that need proper analysis. With a correct interpretation their value would be immeasurable [5such as death or relapse, or a therapeutic response. We recorded descriptive characteristics for all the selected studies. A critical review of outcome-related statistical analyses was undertaken for the articles published in 2004. ResultsNinety studies were identified, and their descriptive characteristics are presented. Sixty-eight (76\%].

Based upon the current knowledge of thyroid cancer three models have been proposed.

\section{MULTISTEP CARCINOGENESIS MODEL}

This is the classic multi-stage model. It is based on the theory that mature thyroid follicular cells can transform into differentiated cells of papillary or follicular thyroid carcinoma and in turn progress to undifferentiated cancer cells characteristic of low-differ- entiation and anaplastic carcinoma [6]. Risk factors such as ionizing radiation can cause genomic instability through direct and indirect mechanisms. They in turn lead to early genetic changes that affect the mitogen-activated protein kinase (MAPK) signaling pathway. The oncogenic activation of MAPK signaling further increases genomic instability, leading to later genetic alterations involving other signaling pathways, cell cycle regulators, and various adhesion molecules [7]. Based on multistep carcinogenesis, follicular carcinomas originate from follicular adenomas and papillary carcinomas from precursor cells, derived from thyrocytes. Anaplastic carcinoma can develop from papillary or follicular thyroid carcinoma by dedifferentiation [8].

\section{FETAL CELL CARCINOGENESIS MODEL}

This model suggests that thyroid carcinoma cells are derived either from normal stem cells or from precursor cells of fetal thyroid cell origin and not from mature thyroid follicular cells [9]. Thus, the model describes the existence of a hierarchy of fetal thyroid cells in the thyroid gland that can lead to various forms of thyroid carcinoma. As a result, thyroid cancer cells derived from this mechanism will have a genetic profile similar to that of fetal thyroid cells [6]. This model identified three separate precursor cells for papillary, follicular and anaplastic thyroid cancer [9]. Prothyrocytes, thyroblasts, and thyroid stem cells are identified by gene expression profiles. There are two major genes: thyroglobulin $(\mathrm{Tg})$ and oncofetal fibronectin (onfFN). Tg positive and onfFN negative cells can be transformed into follicular thyroid carcinoma, $\mathrm{Tg}$ positive and onfFN positive thyroid cells can be transformed into thyroid papillary carcinoma (PTC) and thyroid stem cells that are Tg negative and onfFN positive can be transformed into low-differentiated or anaplastic thyroid carcinoma [9]. It is important to point out that all the articles published in support of this model are from the same researchers.

\section{THYROID CANCER STEM CELLS}

This model is based on the idea that only one subset of cancer cells has the ability to self-renew and produce progenitor cells that replicate and sustain tumor growth [10]. Cancer stem cells can divide symmetrically or asymmetrically and are pluripotent. Therefore, the cancer stem cell model is an attractive way to take into account the functional diversity common in thyroid cancer. CD133 has been used as a marker for cancer stem cells in many types of solid malignancies. CD133 positive cells are thought to be precisely cancer stem cells. In thyroid carcinoma, CD133 posi- 
tive cells were identified in two of the four anaplastic carcinoma cell lines (ARO and KAT-4) [10, 11]. The first in vitro and in vivo experiments were very promising, but in 2008, 20 of 40 cell lines were found to be cross-contaminated with cell lines from other organs. The mentioned before cell lines, ARO and KAT4, were also included in this study and appear to be cross-contaminated with a colon cancer cell line [12].

A number of studies have shown that in tumor material taken during thyroidectomy in patients with follicular, papillary and anaplastic thyroid cancer, there is a small population of aldehyde dehydrogenase (ALDH) positive cells. When these ALDH positive cells are cultured in vitro and in vivo, they form tumors with characteristics comparable to those observed in patients [13]. The existence of thyroid stem cells has important implications for the understanding of the tumor process and the early prognosis of eventual disease progression. This knowledge will help us develop better drug therapies and strategies for treating the disease [6].

At present, it is unclear which of these models, or a combination of these, reflects the true mechanisms of oncogenesis in thyroid cancer [13].

\section{SOMATIC MUTATIONS}

The Cancer Genome Atlas (TCGA) is a joint cancer research program of the National Cancer Institute $(\mathrm{NCl})$ and the National Human Genome Research Institute (NHGRI). The program was proposed in 2005 and started in 2006 with several pilot projects. Its main objective was the overall characterization of somatic changes in the cancer genome. In 2009, the program received additional funding through The American Recovery and Reinvestment Act and expanded its activities to include all common cancers, including thyroid cancer. One of the main goals of the thyroid project was to identify cancer-causing mutations - driver mutations [13]. The results of these studies, published in 2014, confirmed that BRAFV600E was the most common somatic mutation in PTC, whereas mutations in other MAPK-related genes (NRAS, KRAS, and HRAS) were rarer [14]. Other single gene mutations were found in $1-2 \%$ of patients and were thought to be driver mutations in the etiology of thyroid cancer. This fact highlights the importance of rare mutations in tumor development [15]. These heterogeneous genes include translocation-related factor EIF1AX, DNA repair genes CHEK2 and PPM1D, epigenetic regulators MLL (KMT2A), MLL3 (KMT2C), ARID1B and PTEN, AKT1 (2 genes related to the PI3K) AKT signaling pathway, and the PI3K (AKT signaling pathway mutations in the TERT promoter and rare tumor suppressor)cell control related mutations, including TP53, RB1, NF1 and MEN1. This study confirmed and summarized the results from many previous observation studies that 1) classical papillary thyroid carcinoma is rich in BRAF V600E and RET rearrangements, 2) the tallcell variant of papillary carcinoma is characterized by a high frequency of BRAF V600E, and 3) in the follicular variant of papillary carcinoma we have a high frequency of mutations in RAS genes [14].

\section{BRAF}

BRAF is a serine-threonine kinase that travels into the cell membrane after it is bound and activated by RAS, leading to phosphorylation and activation of the MAPK signaling pathway [16]. The BRAF activating mutation provides an alternative pathway for aberrant activation of extracellular signal-regulated kinase (ERK), which is associated with tumorigenesis in several human cancers [7]. The most common mutation in sporadic papillary carcinoma is the BRAF V600E mutation. The follicular variants of papillary carcinoma regularly have the BRAF K601E mutation [15]. In classical papillary carcinomas a VK600-1E deletion (BRAFVK600-1E) has been detected. These facts indicate a possible correlation of genotype-phenotype [15].

The clinical impact of the presence of the BRAF mutation has been extensively investigated. In a recent meta-analysis, the links between BRAF mutation and race, age, sex, size, histologic subtype, extrathyroid growth, metastasis, and stage were examined [1749 (57.6\%]. This study showed that the BRAF mutation in PTC is associated with a specific histologic subtype, extrathyroid invasion, and advanced clinical stage, but not related to the patient's race, age, gender, or tumor size. This suggests that the presence of a BRAF mutation is an important and useful prognostic molecular marker (17)49 (57.6\%. In another study, the BRAF mutation was an independent predictor of tumor recurrence, regardless of the initial stage of the disease - stages I and II [18]. An interesting fact is that the BRAF mutation is associated with a reduced ability to absorb iodine 131 (131I) and failure of treatment in recurrent disease [19-21]. The presence of the BRAF mutation in patients with papillary thyroid carcinoma varies - from 45 to $85 \%$ [22] In anaplastic thyroid carcinoma the incidence is significantly lower than in PTC, about $20-40 \%$ [23]. In the light of the "multistep model of carcinogenesis", this is a significant finding, since according to the model, anaplastic thyroid carcinoma is a dedifferentiated form of PTC and the BRAF mutation is considered an event in 
carcinogenesis. Supporters of other alternative theories use this as an argument against the "multi-step model of carcinogenesis".

A number of research teams have examined the BRAF V600E mutation in fine needle aspiration (FNA) of thyroid tumors with unclear cytologic pattern [20]. The consensus is that testing for BRAF mutation alone in FNA samples is unlikely to resolve the dilemma of undetermined cytology. Only 17 percent of cases with unclear cytology can benefit from the BRAF study [20]. However, recent studies have shown that gene mutations in FNA specimens of PTMC could be successfully analyzed with a higher sensitivity using NGS compared to conventional methods for mutation detection [24, 25RAS, TERT promoter, and TP53 genes in ultrasound-guided fine-needle aspiration (FNA].

\section{RAS}

Human HRAS, KRAS and NRAS genes encode Gproteins that are located on the inner surface of the cell membrane. They transmit signals originating from the tyrosine kinase receptors of the cell membrane to other G-proteins. This is done by using signals by MAPK, PI3K-AKT and others signaling pathways. Thus, they act as a signaling switch regulating cell fate by linking receptor activation to effector pathways to the cell nucleus. In short they regulate cellular processes such as proliferation, differentiation, and cell death [26]. Mutations of RAS genes are frequently observed. The most common mutations are NRAS point mutations in codon 61 and HRAS in codon 61 . The frequency of RAS mutations depends on the type of thyroid cancer - $10-20 \%$ in PTC, in 40$50 \%$ for follicular carcinomas and $20-40 \%$ for poorly differentiated and anaplastic carcinomas [23]. RAS mutations are also found in $20-40 \%$ of benign follicular adenomas. RAS mutations are more common in areas with sufficient iodine and in patients with follicular variant PTC than in classic PTC. The role of RAS mutations in thyroid lesions is controversial. Their presence in adenomas indicates that this may be a precursor to RAS-positive follicular carcinomas and a follicular variant of PTC [23]. The high degree of observed variation in the diagnosis of follicular adenoma and follicular variant PTC also supports this hypothesis [23].

The presence of a RAS mutation may justify the expected malignancy of the disease. There is a close relationship between oncogenic RAS and the loss of histologic features that characterize well-differentiated thyroid tumor phenotypes [27]. RAS mutations are associated with poor prognosis, regardless of tumor stage, and whether the tumor is morphologically classified as low-differentiated, papillary, or follicular [27].

\section{PAX8/PPAR厂 REARRANGEMENT}

The Peroxisome Proliferator Gamma Activator Receptor (PPAR-gamma) is subject to a unique and interesting rearrangement in follicular thyroid carcinoma. PPARy is a member of the nuclear steroid hormone receptor superfamily that forms heterodimers with the retinoid $X$ receptor [28]. The rearrangement results in a fusion between a portion of the PAX8 gene that encodes a double domain transcription factor and the PPARy gene. The fusion results in a strong overexpression of the chimeric PAX8/PPARy protein. It occurs in $30-35 \%$ of patients. Such rearrangements are observed in $2-13 \%$ of the follicular adenomas and in $1-5 \%$ of the follicular variant of PTC [27]. The presence of rearrangements are also associated with an invasive phenotype [28]. RAS mutations and PAX8/ PPARy rearrangements are predominantly present in follicular tumors, but this does not overlap the two mutations. This leads to the conclusion that these conventional follicular thyroid carcinomas consist of at least two distinct groups of tumors that develop through different molecular mechanisms [28]. The signaling pathways involved in the rearrangement of PAX8/PPARy have not yet been fully understood, but several studies have been conducted to profile gene expression and appear to be involved with angiopoietin-4, aquaporin 7, enolase 3 and placental growth factor [29].

\section{RET/PTC REARRANGEMENT}

The RET/PTC rearrangements are a result of fusion between part of the RET gene and one of several possible partner genes. All chimeric genes contain RET, which encodes the intact tyrosine kinase domain of the RET protein, fused to the active promoter of another gene that controls transcription of the RET/ PTC protein [23]. RET/PTC is a classic oncoprotein that activates MAPK and PI3K-AKT pathways. RET/ PTC rearrangements occur with the highest frequency $(50-80 \%)$ in patients exposed to previous ionizing radiation [30]. The prevalence and specificity of RET/ PTC rearrangements in PTC varies dramatically.

In most cases this variation exists because of the heterogeneous distribution of rearrangements in the tumor and the different sensitivity of the detection methods used. RET/PTC rearrangements can be present both in a large number of tumor cells and can be detected by multiple methods (clonal RET/PTC) and in a small cellular groups or individual cells in the 
lesion and thus be detected only by hypersensitivity techniques (non-clonal RET/PTC) [31, 32]. RET/PTC is formed by fusion of the 3 'portion of the RET gene encoding the receptor tyrosine kinase and the 5' portion of various unbound genes. The two most common types of rearrangements, RET/PTC1 and RET/ PTC3, are paracentric inversions, since both RET and its corresponding partner, $\mathrm{H} 4$ or NCOA4, are located on the long arm of chromosome 10. Other known RET/PTC rearrangements are all intrachromosomal translocations [30]. The partner genes have common characteristics: they are expressed in thyroid follicular cells and provide an active promoter for the RET tyrosine kinase domain and contribute to dimerization domains that are essential for dimerization and ligand-independent activation of the shortened RET protein. Virtually all interruptions in the RET gene reside in intron 11, leaving the tyrosine kinase domain of the receptor intact and allowing the RET/PTC proto-oncogene to bind to the SHC via the Y1062 signaling and activate the RAS-RAF-MAPK cascade [33, 34]. RET/PTC1 rearrangements are more common in younger patients. They are less commonly associated with aggressive illness. The clonal rearrangements of RET/PTC are, just like BRAF mutations, $100 \%$ specific for PTC [35].

\section{TRK}

Neurotrophic tyrosine receptor kinase 1 (NTRK1) is another, less commonly detected chromosomal rearrangement occurring in PTC [7]. The NTRK1 gene is located on chromosome 1q22 and can fuse with at least three different partner genes located on the same or different chromosomes - TPM3, TPR and TFG. The activated receptor initiates several signal transduction cascades, including ERK, PI3K and phospholipase-C pathways. It is detected in $5-15 \%$ of sporadic PTC. Unlike other genetic mutations, the frequency of TRK fusions show very large differences in different geographical regions [7, 23].

\section{PTEN}

The protein product of the phosphatase gene and tensin homologue, deleted from chromosome 10 (PTEN) is an important negative regulator of the PI3K/AKT signaling pathway. PTEN is a phosphatase that dephosphorylates PIP3 and thus inhibits the signaling of the PI3K/AKT pathway. Somatic PTEN mutations are also observed in thyroid cancer. The incidence is respectively less than $10 \%$ for follicular carcinomas and between 5 and 15\% for anaplastic cancers [36]. Despite the rarity of this mutation, PTEN function is important in the carcinogenesis of thyroid tumors.

\section{TP53}

TP53 is the gene encoding the cell cycle regulator - p53. Activation of wild-type p53 can lead to cell cycle arrest and the activation of the programed cellular death [16, 23]. This is the normal reaction that prevents the replication of cells with damaged DNA. In contrast, loss of function by mutation in the TP53 induces genomic instability due to attenuated DNA repair systems and subsequent cancer progression. Point mutations are found in $50-80 \%$ of cases of anaplastic thyroid carcinoma. They are less commonly observed in low-differentiated thyroid carcinoma. The frequency of p53 mutations is very low in highly differentiated thyroid carcinoma [37]. These facts contribute significantly to the multistep model of carcinogenesis.

\section{CTNNB1}

TNNB1 is a $\beta$-catenin encoding gene that is involved in cell adhesion and is a downstream effector of the Wnt signaling pathway. Expression on the $\beta$-catenin cell membrane decreases progressively with loss of tumor differentiation. This in turn leads to increased tumor invasiveness and increased metastatic potential [38]. Point mutations in exon 3 of the gene are detected in up to $60 \%$ of anaplastic carcinomas and in $10-20 \%$ of differentiated carcinomas. Analysis of the expression or mutational status of $\beta$-catenin may be useful for typing cancer and effectively predicting the future development of the disease [38].

\section{REGULATORY RNAS}

Recent studies indicate that RNA-mediated regulatory pathways play a role in tumor oncogenesis. Micro-RNA (miRNA, miR) signaling plays a central role in these pathways. Micro-RNAs are short non-coding endogenous RNA fragments that regulate expression at the posttranscriptional level [3937 follicular variant of papillary thyroid carcinoma, and 13 undifferentiated thyroid carcinomas]. To study the role of micro-RNA signaling, Yoruker et al. [40] used RT-PCR testing of serum from patients before and after PTC surgery to measure the level of micro-RNA expression. The serum levels of 4 the 3 miRNAs (miR-222, miR-31, miR-151-5p) were significantly higher while the level of miR-21 was lower in PTC patients than in healthy controls and the. Total levels of all miRNAs were lower in the postoperative samples and showed no significant difference with the healthy control group. A similar study was performed by Lee et al. [41, 42]. They measured the expression of miR-222 and miR$146 \mathrm{~b}$ in plasma and tumor tissues and found elevat- 
ed levels of these mRNAs in recurrent disease. Also, the levels of both markers decreased after thyroidectomy. Thus, their results, especially miR-222 overexpression, confirmed the results of other investigators $[43,44]$. Therefore, we can realistically summarize that both miRNAs can be used as biomarkers to track tumor progression.

MiR-221, miR-22 and miR-21 are involved in the regulation of PTEN (45), while miR-126 is associated with angiogenesis [3937 follicular variant of papillary thyroid carcinoma, and 13 undifferentiated thyroid carcinomas]. Nonetheless, its expression in PTCs as well as in anaplastic thyroid cancer shows a correlation between miR-126 down-regulation and overexpression of VEGF-A mRNA and protein in tumors [3937 follicular variant of papillary thyroid carcinoma, and 13 undifferentiated thyroid carcinomas]. Another study [46] connects downregulation of miR-940, miR$15 \mathrm{a}$ and miR-16 with the manifestation of the PTC phenotype.

\section{MOLECULAR PROFILING OF THYROID CANCER BY NGS}

In recent years, new evidence from a study of somatic mutations in thyroid cancer has been accumulated through the use of targeted next-generation sequencing. Swierniak et al. study used NGS of a panel of 372 genes to investigate the profile of somatic mutations in a total of 82 thyroid follicular tumor tissue samples obtained from 48 Polish patients with thyroid cancer 47 rendering the diagnostics of these tumors challenging. The somatic alterations present in these tumors apart from RAS gene mutations and PAX8/PPARG translocations are not well described. To evaluate the profile of somatic alteration in follicular thyroid tumors, a total of 82 thyroid tissue samples derived from 48 patients were subjected to targeted Illumina HiSeq next generation sequencing of 372 cancer-related genes. New somatic alterations were identified in oncogenes (MDM2, FLI1]. New somatic changes in oncogenes (MDM2, FLI1), transcription factors and repressors (MITF, FLI1, ZNF331), epigenetic enzymes (KMT2A, NSD1, NCOA1, NCOA2) and protein kinases (JAK3, CHEK2, ALK) have been identified. Single nucleotides were most common but major structural variants were rarely found. New DERL/COX6C translocations were detected. Many somatic changes have been observed in non-coding gene regions with high penetrance [47rendering the diagnostics of these tumors challenging. The somatic alterations present in these tumors apart from RAS gene mutations and PAX8/PPARG transloca- tions are not well described. To evaluate the profile of somatic alteration in follicular thyroid tumors, a total of 82 thyroid tissue samples derived from 48 patients were subjected to targeted Illumina HiSeq next generation sequencing of 372 cancer-related genes. New somatic alterations were identified in oncogenes (MDM2, FLI1]. In conclusion, Swierniak et al. summarized that most tumors contain specific somatic changes, suggesting unprecedented heterogeneity [47].

In the next year Zheming Lu et al. published their results [48we identified 11 fusion genes including eight previously reported ones and three novel fusion genes, UEVLD-RET, OSBPL9-BRAF, and SQSTM1NTRK3. Alterations affecting the mitogen-activated protein kinase (MAPK]. They examined 138 specimens of papillary thyroid carcinoma (PTC) for somatic mutation profile and fusion genes by targeted resequencing using a cancer panel (ThyGenCapTM). The panel included 244 cancer-related genes and 20 potential fusions. The results showed at least one genetic alteration (including mutations or fusions) in $85.5 \%$ of the Chinese patients studied. The most commonly mutated gene was BRAF V600E (57.2\%). In addition, the authors were able to identify 11 fusion genes, including eight previously reported and three new fusion genes - UEVLD-RET, OSBPL9-BRAF and SQSTM1-NTRK3, respectively. Changes affecting components of the MAPK signaling pathway were observed in $69.6 \%$ of the PTC cases and all these leading mutations were mutually exclusive. The fusion genes were strongly associated with various clinicopathological characteristics, such as early age, local invasion, extensive metastases, and stage of the disease [48we identified 11 fusion genes including eight previously reported ones and three novel fusion genes, UEVLD-RET, OSBPL9-BRAF, and SQSTM1-NTRK3. Alterations affecting the mitogenactivated protein kinase (MAPK].

All the data presented so far prove that the somatic mutations play an important role in the development of thyroid carcinoma. Despite the leap in our knowledge regarding carcinogenesis in recent years, a number of fundamental questions remain unanswered. Elucidating new rare somatic mutations and genomic rearrangements can improve preoperative diagnosis and optimize post-operative strategies for the treatment of thyroid cancer $[25,49$ most frequently Bethesda category III or IV. Diagnostic surgeries can be avoided for these patients if the nodules are reliably diagnosed as benign without surgery. Objective: To determine the diagnostic accuracy of a multigene classifier (GC]. NGS enables the tracking of mutations in a large set of genes as well as other 
genomic changes related to oncogenesis $[50,51$ the Afirma Gene Sequencing Classifier (GSC] Based on the advances in the NGS technology and the discovery of new thyroid cancer biomarkers molecular testing for indetermined thyroid nodules (ThyroSeq and others) has been developed and is widely used as an additional diagnostic tool for thyroid nodules [50, 51the Afirma Gene Sequencing Classifier (GSC].

Disclosure summary: The authors have nothing to disclose.

\section{REFERENCES:}

1. Eng C. Familial Papillary Thyroid Cancer - Many Syndromes, Too Many Genes? J Clin Endocrinol Metab [Internet]. 2000;85(5):1755-7. Available from: http://press.endocrine. org/doi/abs/10.1210/jcem.85.5.6632

2. Jindrichova S, Vicek P, Bendlova B. [Genetic causes of the thyroid carcinomas]. Cas Lek Ces [Internet]. 2004;143(10):664-8. Available from: http://www.ncbi.nlm.nih. gov/pubmed/15584614

3. Moses W, Weng J, Kebebew E. Prevalence, clinicopathologic features, and somatic genetic mutation profile in familial versus sporadic nonmedullary thyroid cancer. Thyroid [Internet]. 2011;21(4):367-71. Available from: http://www.ncbi.nlm.nih. gov/pubmed/21190444

4. Rios A, Rodriguez JM, Navas D et al. Family Screening in Familial Papillary Carcinoma: The Early Detection of Thyroid Disease. Ann Surg Oncol. 2016/03/30. 2016;23(8):2564-70.

5. Dupuy A, Simon RM. Critical Review of Published Microarray Studies for Cancer Outcome and Guidelines on Statistical Analysis and Reporting. JNCl J Natl Cancer Inst [Internet]. 2007;99(2):147-57. Available from: http://dx.doi.org/10.1093/ jnci/djk018

6. Lin R-Y. Thyroid cancer stem cells. Nat Rev Endocrinol [Internet]. 2011;7:609. Available from: http://dx.doi.org/10.1038/ nrendo.2011.127.

7. Kondo T, Ezzat S, Asa SL. Pathogenetic mechanisms in thyroid follicular-cell neoplasia. Nat Rev Cancer. 2006;6(4):292.

8. Parameswaran R, Brooks S, Sadler GP. Molecular pathogenesis of follicular cell derived thyroid cancers. Int $\mathrm{J}$ Surg. 2010;8(3):186-93.

9. Takano T. Fetal cell carcinogenesis of the thyroid: theory and practice. In: Seminars in cancer biology. Elsevier; 2007. p. 233-40.

10. Friedman S, Lu M, Schultz A et al. CD133+ anaplastic thyroid cancer cells initiate tumors in immunodeficient mice and are regulated by thyrotropin. PLoS One. 2009;4(4):e5395.

11. Zito G, Richiusa P, Bommarito $A$ et al. In vitro identification and characterization of CD133pos cancer stem-like cells in anaplastic thyroid carcinoma cell lines. PLoS One. 2008;3(10):e3544.

12. Schweppe RE, Klopper JP, Korch C et al. Deoxyribonucleic acid profiling analysis of 40 human thyroid cancer cell lines reveals cross-contamination resulting in cell line redundancy and misidentification. J Clin Endocrinol Metab. 2008;93(11):4331-41.

13. Todaro M, lovino F, Eterno $V$ et al. Tumorigenic and metastatic activity of human thyroid cancer stem cells. Cancer Res. 2010;70(21):8874-85.
14. Giordano TJ. The Cancer Genome Atlas Research Network: A Sight to Behold. Endocr Pathol [Internet]. 2014 Dec;25(4):362-5. Available from: https://doi.org/10.1007/ s12022-014-9345-4

15. Lawrence: Mutational heterogeneity in cancer and... - Google Наука [Internet]. [cited 2020 Jan 2]. Available from: https:// scholar.google.com/scholar_lookup?title=Mutational heterogeneity in cancer and the search for new cancer-associated genes\&author=MS. Lawrence\&author=P. Stojanov\&author=P. Polak\&journal $=$ Nature $\&$ volume $=499 \&$ pages $=214$ 218\&publication_year $=2013$

16. Nikiforova MN, Nikiforov YE. Molecular genetics of thyroid cancer: implications for diagnosis, treatment and prognosis. Expert Rev Mol Diagn. 2007/12/20. 2008;8(1):83-95.

17. Ahn HY, Chung YJ, Kim BS et al. Clinical significance of the BRAF V600E mutation in multifocal papillary thyroid carcinoma in Korea. Ahn HY, Chung YJ, Kim BS, Kang KH, Seok JW, Kim HS, et al., editors. Surgery [Internet]. 2014;155(4):68995. Available from: http://www.ncbi.nlm.nih.gov/entrez/ query.fcgi?cmd=Retrieve\&db=PubMed\&dopt=Citation\&li st_uids $=24612623$

18. Xing $M$, Westra $W H$, Tufano RP et al. BRAF mutation predicts a poorer clinical prognosis for papillary thyroid cancer. J Clin Endocrinol Metab. 2005;90(12):6373-9.

19. Xing M, Alzahrani AS, Carson KA et al. Association between BRAF V600E mutation and mortality in patients with papillary thyroid cancer. Jama. 2013/04/11. 2013;309(14):1493-501.

20. Xing M. BRAF mutation in thyroid cancer. Endocr Relat Cancer. 2005;12(2):245-62.

21. Kim TH, Park YJ, Lim JA et al. The association of the BRAF(V600E) mutation with prognostic factors and poor clinical outcome in papillary thyroid cancer: a meta-analysis. Cancer. 2011/09/02. 2012;118(7):1764-73.

22. Kim KH, Kang DW, Kim SH et al. Mutations of the BRAF gene in papillary thyroid carcinoma in a Korean population. Yonsei Med J. 2004;45:818-21.

23. Nikiforov YE, Nikiforova MN. Molecular genetics and diagnosis of thyroid cancer. Nat Rev Endocrinol. 2011/09/01. 2011;7(10):569-80.

24. Kouba E, Ford A, Brown CG et al. Detection of BRAF V600E Mutations With Next-Generation Sequencing in Infarcted Thyroid Carcinomas After Fine-Needle Aspiration. Am J Clin Pathol [Internet]. 2018 Jul 3 [cited 2020 Jan 5];150(2):17785. Available from: https://academic.oup.com/ajcp/article/150/2/177/5032503

25. Yu FX, Hu MX, Zhao HX et al. Precise Detection of Gene Mutations in Fine-Needle Aspiration Specimens of the Papillary Thyroid Microcarcinoma Using Next-Generation Sequencing. Int J Endocrinol. 2019;2019.

26. Couto JP, Prazeres H, Castro P et al. How molecular pathology is changing and will change the therapeutics of patients with follicular cell-derived thyroid cancer. J Clin Pathol. 2009;62(5):414-21.

27. Garcia-Rostan G, Zhao H, Camp RL et al. Ras mutations are associated with aggressive tumor phenotypes and poor prognosis in thyroid cancer. J Clin Oncol. 2003;21(17):3226-35.

28. Lacroix L, Lazar V, Michiels $S$ et al. Follicular thyroid tumors with the PAX8-PPARY1 rearrangement display characteristic genetic alterations. Am J Pathol. 2005;167(1):223-31.

29. Nikiforova MN, Lynch RA, Biddinger PWet al. RAS point mutations and PAX8-PPARY rearrangement in thyroid tumors: evidence for distinct molecular pathways in thyroid follicular carcinoma. J Clin Endocrinol Metab. 2003;88(5):2318-26.

30. Gandhi M, Evdokimova V, Nikiforov YE. Mechanisms of chromosomal rearrangements in solid tumors: the mod- 
el of papillary thyroid carcinoma. Mol Cell Endocrinol. 2010;321(1):36-43.

31. Unger K, Zitzelsberger $\mathrm{H}$, Salvatore $\mathrm{G}$ et al. Heterogeneity in the distribution of RET/PTC rearrangements within individual post-Chernobyl papillary thyroid carcinomas. J Clin Endocrinol Metab. 2004;89(9):4272-9.

32. Zhu Z, Ciampi R, Nikiforova MN et al. Prevalence of RET/ PTC rearrangements in thyroid papillary carcinomas: effects of the detection methods and genetic heterogeneity. J Clin Endocrinol Metab. 2006;91(9):3603-10.

33. Ciampi R, Nikiforov YE. RET/PTC rearrangements and BRAF mutations in thyroid tumorigenesis. Endocrinology. 2007;148(3):936-41.

34. Knauf JA, Kuroda H, Basu S, Fagin JA. RET/PTC-induced dedifferentiation of thyroid cells is mediated through $Y 1062$ signaling through SHC-RAS-MAP kinase. Oncogene. 2003;22(28):4406.

35. Witt RL, Ferris RL, Pribitkin EA et al. Diagnosis and management of differentiated thyroid cancer using molecular biology. Laryngoscope. 2013;123(4):1059-64.

36. Xing M. Genetic alterations in the phosphatidylinositol-3 kinase/ Akt pathway in thyroid cancer. Thyroid. 2010;20(7):697-706.

37. Nikiforova MN, Wald Al, Roy S et al. Targeted next-generation sequencing panel (ThyroSeq) for detection of mutations in thyroid cancer. J Clin Endocrinol Metab. 2013;98(11):E1852-60.

38. Garcia-Rostan G, Camp RL, Herrero A et al. $\beta$-catenin dysregulation in thyroid neoplasms: down-regulation, aberrant nuclear expression, and CTNNB1 exon 3 mutations are markers for aggressive tumor phenotypes and poor prognosis. Am J Pathol. 2001;158(3):987-96.

39. Salajegheh A, Vosgha $H$, Rahman MA et al. Interactive role of miR-126 on VEGF-A and progression of papillary and undifferentiated thyroid carcinoma. Hum Pathol. 2016 May 1;51:75-85.

40. Yoruker EE, Terzioglu D, Teksoz S et al. MicroRNA expression profiles in papillary thyroid carcinoma, benign thyroid nodules and healthy controls. J Cancer. 2016;7(7):803-9.

41. Lee JC, Zhao JT, Clifton-Bligh RJ et al. MicroRNA-222 and MicroRNA-146b are tissue and circulating biomarkers of recurrent papillary thyroid cancer. Cancer. $2013 \mathrm{Dec}$ 15;119(24):4358-65.

42. Xiang D, Tian B, Yang T, Li Z. miR-222 expression is correlated with the ATA risk stratifications in papillary thyroid carcinomas. Medicine (Baltimore). 2019 Jun 1;98(25):e16050.
43. Pallante P, Battista S, Pierantoni GM, Fusco A. Deregulation of microRNA expression in thyroid neoplasias. Pallante P, Battista S, Pierantoni GM, Fusco A, editors. Nat Rev Endocrinol [Internet]. 2013/11/20. 10(2):88-101. Available from: http://www.ncbi.nlm.nih.gov/entrez/query.fcgi?cmd=Retrieve $\& \mathrm{db}=$ PubMed\&dopt $=$ Citation\&list_uids $=24247220$

44. He H, Jazdzewski K, Li W et al. The role of microRNA genes in papillary thyroid carcinoma. Proc Natl Acad Sci U S A. 2005 Dec 27;102(52):19075-80.

45. Zhang J, Wang J, Zhao F et al MicroRNA-21 (miR-21) represses tumor suppressor PTEN and promotes growth and invasion in non-small cell lung cancer (NSCLC). Clin Chim Acta [Internet]. 2010 Jun 3 [cited 2020 Jan 5];411(1112):846-52. Available from: http://www.ncbi.nlm.nih.gov/ pubmed/20223231

46. Hu J, Li C, Liu C et al. Expressions of miRNAs in papillary thyroid carcinoma and their associations with the clinical characteristics of PTC. Cancer Biomark [Internet]. 2017 [cited 2020 Jan 5];18(1):87-94. Available from: http://www.ncbi.nlm.nih. gov/pubmed/28085013

47. Swierniak M, Pfeifer A, Stokowy T et al. Somatic mutation profiling of follicular thyroid cancer by next generation sequencing. Mol Cell Endocrinol [Internet]. 2016 Sep 15 [cited 2020 Jan 2];433:130-7. Available from: https://www.sciencedirect. com/science/article/pii/S0303720716302076

48. Lu Z, Zhang Y, Feng D et al. Targeted next generation sequencing identifies somatic mutations and gene fusions in papillary thyroid carcinoma. Oncotarget. 2017;8(28):45784-92.

49. Nikiforov YE, Steward DL, Carty SE et al. Performance of a Multigene Genomic Classifier in Thyroid Nodules with Indeterminate Cytology: A Prospective Blinded Multicenter Study. JAMA Oncol. 2019 Feb 1;5(2):204-12.

50. Nikiforov YE, Baloch ZW. Clinical validation of the ThyroSeq v3 genomic classifier in thyroid nodules with indeterminate FNA cytology. Vol. 127, Cancer Cytopathology. John Wiley and Sons Inc.; 2019. p. 225-30.

51. Nicholson KJ, Roberts MS, McCoy KL et al. Molecular Testing Versus Diagnostic Lobectomy in Bethesda III/IV Thyroid Nodules: A Cost-Effectiveness Analysis. Thyroid. 2019 Sep 1;29(9):1237-43.

Received: 05 February 2020, Accepted: 14 February 2020 